\title{
Schottky Barriers in Carbon Nanotube Heterojunctions
}

\author{
Arkadi A. Odintsov ${ }^{1,2}$ \\ ${ }^{1}$ Department of Applied Physics and DIMES, Delft University of Technology, 2628 CJ Delft, The Netherlands \\ ${ }^{2}$ Nuclear Physics Institute, Moscow State University, Moscow 119899 GSP, Russia
}

(Received 11 October 1999)

\begin{abstract}
Electronic properties of heterojunctions between metallic and semiconducting single-wall carbon nanotubes are investigated. Ineffective screening of the long-range Coulomb interaction in one-dimensional nanotube systems drastically modifies the charge transfer phenomena compared to conventional semiconductor heterostructures. The length of depletion region varies over a wide range sensitively depending on the doping strength. The Schottky barrier gives rise to an asymmetry of the $I-V$ characteristics of heterojunctions, in agreement with recent experimental results by Yao et al. and Fuhrer et al. Dynamic charge buildup near the junction results in a steplike growth of the current at reverse bias.
\end{abstract}

PACS numbers: 71.20.Tx, 73.23. $-\mathrm{b}, 73.30 .+\mathrm{y}$

Single-wall carbon nanotubes (SWNTs) are giant linear fullerene molecules which can be studied individually by methods of nanophysics [1]. Depending on the wrapping of a graphene sheet, SWNTs can either be one-dimensional (1D) metals or semiconductors with the energy gap in subelectronvolt range [2,3]. While metallic nanotubes can play a role of interconnects in future electronic circuits, their semiconducting counterparts can be used as basic elements of switching devices. An example is the field effect transistor on semiconducting SWNT operating at room temperature [4].

Of particular interest are all-nanotube devices [5]. The simplest can be fabricated by contacting two SWNTs with different electronic properties. The SWNTs can be seamlessly joined together by introducing topological defects (pentagon-heptagon pairs) into the hexagonal graphene network [6]. The resulting on-tube junction generically has the shape of a kink. Electronic properties of such junctions have been investigated theoretically (see, e.g., Refs. [7] and [8]) within the model of noninteracting electrons.

Electron transport in nanotube heterojunctions has been studied in two recent experiments. Yao et al. treated junctions in SWNTs with kinks [9] whereas Fuhrer et al. explored contacts of crossed nanotubes [10]. Both groups observed nonlinear and asymmetric $I-V$ characteristics resembling that of rectifying diodes. On one hand, the rectifying behavior can be naturally interpreted in terms of Schottky barriers (SBs). On the other hand, formation of a SB might be surprising since one expects no charge transfer in junctions between two SWNTs made of the same material.

A possible reason for the charge transfer might be the doping of the nanotubes forming the heterojunction [11]. The doping can be caused by introduction of dopant atoms into the nanotubes or by charge transfer from metallic electrodes. In the latter case the doping strength can also be controlled by the gate voltage. It is important to mention that screening of the Coulomb interaction is ineffective in one-dimensional nanotubes. For this reason the effect of the doping is long ranged: the density of the transferred charge decays slowly with the distance from the electrodes and might be appreciable at the heterojunction [12].

The long-range Coulomb interaction should be properly taken into account when treating the charge transfer in the heterojunction itself. Unfortunately, this was not accomplished in Ref. [11], where the electric field was assumed to be fully screened in the region of a few atomic layers near the junction. In this Letter, we study charge transfer phenomena in nanotube heterojunctions with true long-range Coulomb interaction. We concentrate on the metal-semiconductor SWNT junction and analyze its equilibrium and nonequilibrium properties (SB parameters, $I-V$ characteristics) by solving the Poisson equation self-consistently.

As a model system we consider "straight" junction [13] between metallic $(x<0)$ and semiconducting $(x>0)$ SWNTs (Fig. 1). We assume that the conducting $p_{z}$ electrons in SWNTs are confined to the surface of a cylinder of radius $R$. The nanotubes are surrounded by a coaxial cylindrical gate electrode of radius $R_{s} \gg R$. The Fourier components of the 1D Coulomb interaction are given by

$$
U_{q}=\frac{2 e^{2}}{\kappa}\left\{I_{0}(q R) K_{0}(q R)-\frac{I_{0}^{2}(q R) K_{0}\left(q R_{S}\right)}{I_{0}\left(q R_{S}\right)}\right\},
$$

with the dielectric constant of the medium $\kappa$ and the modified Bessel functions $I_{0}, K_{0}$. Equation (1) describes the

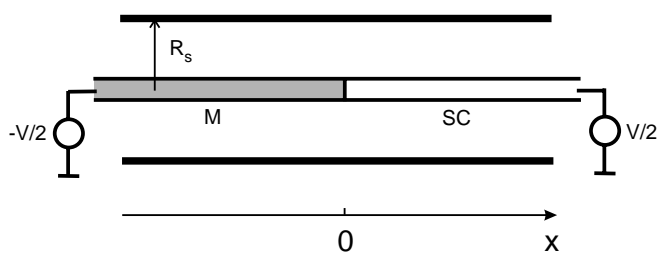

FIG. 1. Heterojunction between metallic (M) and semiconducting (SC) nanotubes. The potential $V_{g}$ is applied to a cylindrical gate electrode of radius $R_{s}$. 
long-range Coulomb interaction, $U(x)=1 / \kappa x$, for $R \ll$ $x \ll R_{s}$. The interaction is screened at large distances $x \gg R_{s}$, so that $U_{0}=e^{2} / C=\left(2 e^{2} / \kappa\right) \ln \left(R_{S} / R\right), C$ being the capacitance of SWNT per unit length. The kernel (1) relates the electrostatic potential $\varphi$ at the surface of SWNTs to $1 \mathrm{D}$ charge density $e \rho(e>0)$,

$$
e \varphi_{q}=U_{q} \rho_{q} .
$$

Since experimental values $[9,10]$ of the conductance of heterojunctions are small, $G /\left(e^{2} / h\right) \lesssim 10^{-2}$, we will assume low transparency $T \ll 1$ of the barrier between two SWNTs. In this case the electrons in the nanotubes are described by the equilibrium Fermi distribution $f(E)$, also when the voltage $V$ is applied to the system.

In equilibrium, the charge density is related to the energy $\tilde{E}_{0}(x)=E_{0}(x)-E_{F}(x)$ of the gapless point (charge neutrality level) of graphite $E_{0}$ counted from the Fermi level $E_{F}$,

$$
\rho(x)=\int d E \operatorname{sgn}(E) \nu(E) f\left\{\left[E-\tilde{E}_{0}(x)\right] \operatorname{sgn}(E)\right\},
$$

with the density of electronic states $\nu$ [14]. Equation (3) is valid provided that $\tilde{E}_{0}(x)$ varies slowly on the scale of the Fermi wavelength.

We will neglect the effect of higher 1D subbands in SWNTs. This is legitimate at low energies, $\left|\tilde{E}_{0}\right|<\Delta^{(1)}$ and $k_{B} T \ll \Delta^{(1)}$, where $\Delta^{(1)} /\left(\hbar v_{F} / R\right)=1(2 / 3)$ for metallic (semiconducting) SWNT. The densities of states in metallic and semiconducting SWNTs are given by

$$
\nu_{M}=\frac{4}{\pi \hbar v_{F}}, \quad \nu_{S}=\frac{4}{\pi \hbar v_{F}} \frac{|E| \Theta(|E|-\Delta)}{\sqrt{E^{2}-\Delta^{2}}},
$$

with the Fermi velocity $v_{F} \simeq 8.1 \times 10^{5} \mathrm{~m} / \mathrm{s}$ and the energy gap $2 \Delta=2 \hbar v_{F} / 3 R$ in semiconducting $\operatorname{SWNT}(\Delta \simeq$ $0.3 \mathrm{eV}$ for generic SWNTs [2] with $R=0.5-0.7 \mathrm{~nm})$.

In the limit of zero temperature Eq. (3) may be inverted as

$$
\tilde{E}_{0}(\rho)= \begin{cases}\rho / \nu_{M}, & x<0, \\ \sqrt{\Delta^{2}+\left(\rho / \nu_{M}\right)^{2}} \operatorname{sgn}(\rho), & x>0 .\end{cases}
$$

The charge neutrality level $\tilde{E}_{0}(x)$ is related to the electrostatic potential (2),

$$
\tilde{E}_{0}(x)+e \varphi(x)=\mu+e V \operatorname{sgn}(x) / 2,
$$

$\mu \mp e V / 2$ being the electrochemical potentials for holes in metallic and semiconducting SWNTs. The potential $\mu=\alpha\left(\Delta W-e V_{g}\right)$ can be controlled by the gate voltage $V_{g}$ (Fig. 1). It also incorporates the difference $\Delta W=$ $W_{M}-W_{N T}$ of the work functions of the gate electrode and SWNT [15] (the coefficient $\alpha$ characterizes mutual capacitance of the nanotubes to the gate and is equal to unity in our case).

We solve Eqs. (2), (3), and (6) self-consistently by numerical minimization of the corresponding energy functional. The Coulomb energy is computed in the Fourier space. Figures 2 and 3 display the results for the following parameters: $R_{S} / R=75$ and $\nu_{M} U_{0} / \ln \left(R_{S} / R\right)=5$. The
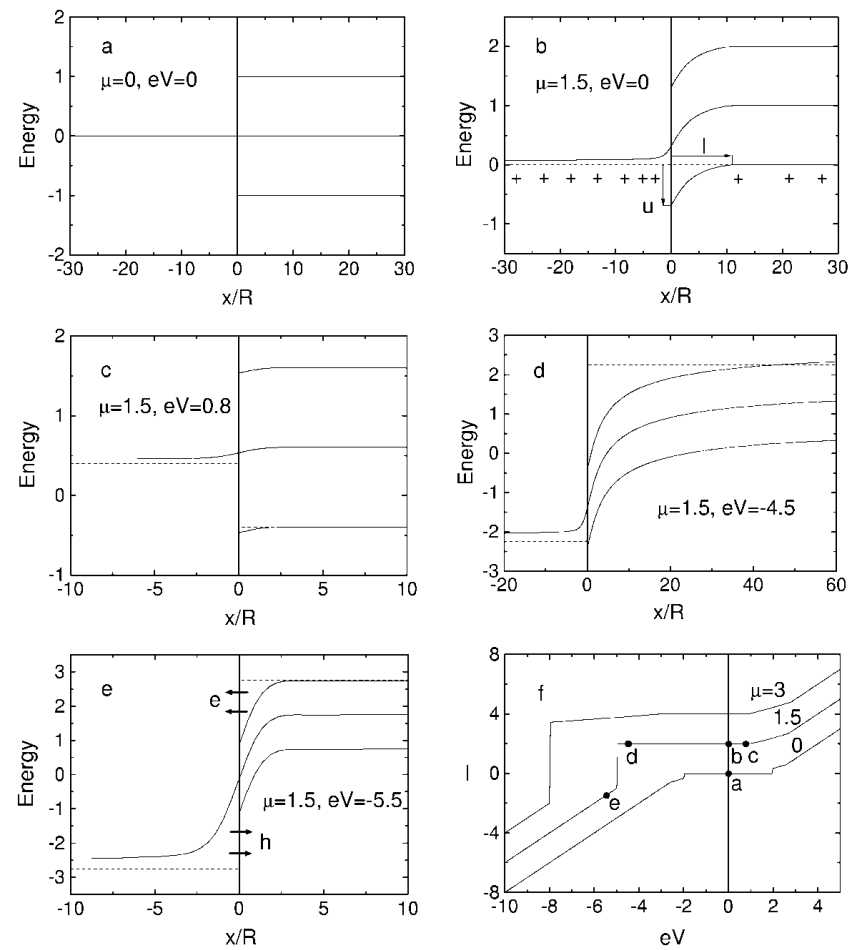

FIG. 2. The charge neutrality level $\bar{E}_{0}$ and the energies of the conduction $E_{c}$ and valence $E_{v}$ bands as functions of the distance from the junction (a)-(e). The Fermi levels are shown by dashed lines. The $I-V$ characteristics of the heterojunction at zero temperature (f). All the energies $\left(\bar{E}_{0}, E_{c}, E_{v}, \mu, e V\right)$ are in units of $\Delta$; the current is in units of $2 e \Delta T_{i} /(\pi \hbar)$. The $I-V$ curves for $\mu=1.5,3$ are offset for clarity.

latter value corresponds to the dielectric constant $\kappa \simeq 1.4$ which can be inferred from the experimental data (see Fig. 4 of Ref. [1]).

The band bending diagrams (Fig. 2) display the charge neutrality level $\bar{E}_{0}(x)=\tilde{E}_{0}(x)-e V \operatorname{sgn}(x) / 2$ counted from the "average" Fermi level of metallic and

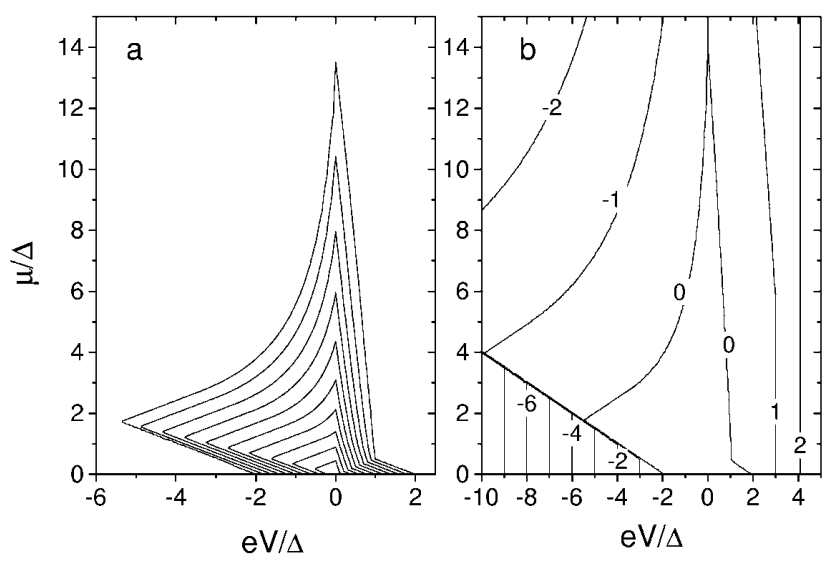

FIG. 3. The height $u$ of the Schottky barrier (a) and the current $I$ through the heterojunction (b) at zero temperature as functions of the electrochemical potential $\mu$ and bias voltage $V$. The contour lines (a) correspond to $u / \Delta=0,0.1, \ldots, 0.9$ from periphery to origin. The energies $\mu, e V$ are in units of $\Delta$. The current is in units of $2 e \Delta T_{i} /(\pi \hbar)$. 
semiconducting SWNTs, as well as the energies $E_{c, v}=$ $\bar{E}_{0} \pm \Delta$ of the conduction and valence bands in semiconducting SWNT.

Let us start from the case of zero bias, $V=0$ [Figs. 2(a) and 2(b)]. At zero electrochemical potential, $\mu=0$, the Fermi level of the nanotubes coincides with the gapless point of graphite and the system is charge neutral [Fig. 2(a)]. This situation occurs for isolated nanotubes. The barriers for the electron and hole transport are equal to $\Delta$.

To make contact with the experiments $[9,10]$ we will concentrate on $p$-doped SWNTs $(\mu>0)$. Because of a larger number of electronic states $\int_{0}^{\tilde{E}_{0}} d E \nu(E)$ the metallic SWNT acquires more charge and has higher electrostatic potential $\varphi(-\infty)$ [lower charge neutrality level $\tilde{E}_{0}(-\infty)$ ] compared to semiconducting SWNT kept at the same electrochemical potential; see Eqs. (5) and (6). The electric field induced by this charge bends the bands in the semiconducting SWNT downwards so that a SB is formed near the interface [Fig. 2(b)].

For $|\mu|<\Delta$, there are no free charges in the semiconducting SWNT. Our numerical results indicate that the electrostatic potential $\varphi(x)$ decays logarithmically at $R \ll$ $x \ll R_{s}$ so that the band bending extends over long distances $x \sim R_{s}$ [the analytical estimate, $\varphi(x) \simeq e \nu_{M} \mu \times$ $\ln \left(R_{S} / x\right) / \kappa$, is available in the limit of weak interaction, $\left.\nu_{M} U_{0} \ll 1\right]$. At $\mu=\Delta$ holes enter the semiconducting SWNT. With increasing the electrochemical potential the holes come closer to the junction reducing the length $l$ and the height $u$ of a SB [Fig. 2(b)]. In the case of weakly doped semiconducting SWNT, $\mu=\Delta(1+\delta), \delta \ll 1$, a rough estimate of the depletion length $l$ can be made, $\ln \left(l / R_{s}\right) \sim \delta \ln \left(R / R_{s}\right)$, for $R \ll l \ll R_{s}$. Therefore, the depletion length changes rapidly from $l \sim R_{s}$ to $l \sim R$ with increasing doping in this regime. The height of a SB can be estimated from the difference of the charge neutrality levels in semiconducting and metallic SWNTs, $u \lesssim$ $\tilde{E}_{0}(\infty)-\tilde{E}_{0}(-\infty)$. The latter evaluate at $\tilde{E}_{0}(-\infty)=\mu /$ $\left(1+\nu_{M} U_{0}\right)$ and $\tilde{E}_{0}(\infty)=\Delta$ for $\delta \ll \nu_{M} U_{0}$; see Eqs. (2), (5), and (6). Since the band bending occurs predominantly in the semiconducting part [Figs. 2(a) and 2(b)] and $\nu_{M} U_{0} \gg 1$, one expects that $u \simeq \Delta$ for $\delta \ll \nu_{M} U_{0}$.

Figure 3(a) shows the result for the SB height $u$ defined as the minimum energy of electron or hole excitation required to transfer the elementary charge across the junction in the absence of tunneling through the SB. The SB height shows pronounced asymmetry as a function of the bias voltage. At small electrochemical potential the SB height at forward (reverse) bias is determined by the energy of the valence (conduction) band in the "bulk" of semiconducting SWNT, $x \rightarrow \infty$, with respect to the Fermi level, $u_{f(r)}^{(b)}=$ $\Delta \mp \tilde{E}_{0}(\infty)$, with $\tilde{E}_{0}(\infty)=\mu+e V / 2$ [Fig. 2(d)]. This corresponds to straight portions of contour lines in the lower part of Fig. 3(a). In particular, the positive $V_{+}$and negative $V_{-}$threshold voltages at which the SB vanishes $\left(u_{f(r)}^{(b)}=0\right)$ are given by $e V_{ \pm}^{(b)}= \pm 2 \Delta-2 \mu$.
Straight portions of contour lines [Fig. 3(a)] are interrupted by cusps. At forward bias the cusps occur along the line $e V=2 \mu$ [Fig. 3(a)] where the charge density in metallic SWNT and the band bending change sign; see Eq. (6). Above the point of a cusp, the height of a SB at forward (reverse) bias corresponds to the energy of the valence band at the interface of SWNTs, $x=0$, counted from the Fermi level of semiconducting (metallic) nanotube, $u_{f(r)}^{(i)}=\Delta-\tilde{E}_{0}( \pm 0)$ [Fig. 2(c)]. The threshold voltages $V_{+(-)}^{(i)}$, correspond to suppression of a SB at the interface, $u_{f(r)}^{(i)}=0$. Note that at $\mu \geq \Delta / 2$ the positive threshold $e V_{+}^{(i)} \simeq \Delta$ is relatively insensitive to the electrochemical potential, Fig. 3(a). This can be used for a rough estimate of the gap from experimental data.

We will proceed with the analysis of nonequilibrium electron transport. The current through the heterojunction is given by the Landauer formula,

$$
I=\frac{2 e}{\pi \hbar} \int d E T(E)\{f(E-e V / 2)-f(E+e V / 2)\}
$$

with the energy-dependent transmission coefficient $T(E)$ of the junction. It is natural to separate the contribution $T_{i}(E)$ of a barrier at the interface between SWNTs [8] and the contribution $T_{S}(E)$ of a SB to the total transmission. As a minimal model, we assume that the transparency $T_{i}$ is energy independent, whereas the transparency $T_{S}(E)$ increases from zero to unity when the energy $E$ crosses the edge of a SB. In this case the total transmission reads $T(E)=0,\left(T_{i}\right)$, for the energies in (out of) the SB range $\left[E_{\min }, E_{\max }\right]$. In the case of downward bending (Fig. 2) the SB range is given by $\left[E_{v}(0), E_{c}(\infty)\right]$, in the absence of charge carriers in the conduction band, $\mu+e V / 2>-\Delta$ [Figs. 2(a)-2(d)], and by $\left[E_{v}(0), E_{c}(0)\right]$, in their presence, $\mu+e V / 2<-\Delta$ [Fig. 2(e)]. The results for the $I-V$ characteristics at zero temperature are presented in Figs. 2(f) and 3(b).

At low electrochemical potential the current increases abruptly at positive $V_{+}^{(b)}$ and negative $V_{-}^{(b)}$ threshold voltages which correspond to inclined straight lines in the lower part of Fig. 3(b) [see also Fig. 2(f)]. At the positive (negative) threshold, $u_{f(r)}^{(b)}=0$, the electrons enter the valence (conduction) band in the bulk of semiconducting SWNT. This causes charge buildup near the junction which, in turn, leads to the reconstruction of the band profile [cf. Figs. 2(d) and 2(e)]. The reconstruction results in the onset of the hole and electron (at reverse bias) channels of transport [Fig. 2(e)] giving rise to a steplike growth of the current.

At higher electrochemical potential the onset of the current at the thresholds $V_{+}^{(i)}, V_{-}^{(i)}$ occurs gradually [Figs. 2(f) and 3(b)] due to opening of the hole transport channel [Fig. 2(c)]. The cusp at the $I-V$ characteristics [Fig. 2(f)] at somewhat higher voltages $\left(V>V_{+}^{(i)}\right)$ corresponds to the 
onset of the electron channel. Note that at high (forward or reverse) bias both the electron and hole channels are open so that the current is given by $I=\left(2 e T_{i} / \pi \hbar\right)[e V-$ $2 \Delta \operatorname{sgn}(V)]$.

We now consider quantum tunneling through a SB. The transparency $T_{S}(E)$ of a SB can be evaluated using the WKB method and the effective mass approximation. For a triangular barrier of the length $l$ and the height $u$ we obtain

$$
T_{S} \sim \exp \left[-\frac{4 l}{9 R} \sqrt{\frac{2 u}{\Delta}}\right] .
$$

The transparency $T_{S}$ increases considerably near the boundaries of the transport blockade region $\left[V_{-}, V_{+}\right]$ [Fig. 3(a)] due to decreasing $u$ and $l$. For example, $T_{S} \sim$ $2.5 \times 10^{-3}$ for the SB in Fig. 2(b), whereas $T_{S} \sim 1$ for the SBs in Figs. 2(c) and 2(d). This gives rise to a substantial leakage current in the blockade region.

The asymmetry of the $I-V$ characteristics and threshold voltages has been discovered in recent experiments $[9,10]$. According to the data of Ref. [9], both the thresholds $V_{+}$, $V_{-}$shift upwards with the gate voltage. Moreover, the positive threshold shifts less than the negative one. Such behavior is consistent with our model in the regime of moderate doping, $0.5<\mu / \Delta \lesssim 1.8$ (Fig. 3). However, the blockade region of 3-4 $\mathrm{V}$ detected in the experiment is somewhat wider than the theoretical estimate, $V_{+}-$ $V_{-} \lesssim 6.5 \Delta \simeq 2 \mathrm{eV}$. The extra voltage drop could be due to potential disorder in semiconducting SWNT [16] and/or an additional SB at the interface between semiconducting SWNT and metallic electrode.

We now check the model against the experimental data of Ref. [10]. The measured width of the blockade region, $0.5-0.7 \mathrm{~V}$, agrees with the theoretical estimate. The gap in semiconducting SWNT, $\Delta \simeq e V_{+}$, evaluates at $\Delta=0.19$, $0.29 \mathrm{eV}$ for the two devices studied [10]. These values are in the expected range $\Delta \sim 0.25-0.35 \mathrm{eV}[2,3]$. A smooth onset of the current over the range $\sim 0.1-0.3 \mathrm{eV}$ around threshold voltages is naturally associated with quantum tunneling through a "leaky" SB (thermal energies are much smaller, $k_{B} T \simeq 5 \mathrm{meV}$ ). Finally, the steplike feature of the current under reverse bias almost certainly corresponds to the reconstruction of the band profile due to the Fermi level entering the conduction band of semiconducting SWNT. Gradual onset of the differential conductance following the reconstruction might be associated with increasing conductance of disordered semiconducting SWNT under the doping [16].

To conclude, we have studied the electronic properties of carbon nanotube heterojunctions and provided explanation for the main features of recent experimental data $[9,10]$. Because of the long-range Coulomb interaction, the charge transfer phenomena in one-dimensional nanotube systems differ drastically from those in conventional semiconductor heterostructures. This creates new challenges in the design of novel electronic devices. In particular, the long-range electrostatic potential in underdoped junctions might affect other components of a circuit, whereas substantial leakage current in overdoped junctions spoils the rectification. In view of these challenges a new concept of functional devices on molecular level might be needed.

In the process of writing this paper I became aware of the preprint by Léonard and Tersoff [17] who investigated equilibrium properties of junctions between semiconducting SWNTs and found the long-range charge-transfer phenomena in these systems (see also Ref. [12]).

The author wishes to thank B.L. Altshuler, G.E.W. Bauer, Yu. V. Nazarov, S. Tarucha, Y. Tokura, Z.Yao, and, especially, C. Dekker and P. McEuen for stimulating discussions. F. Léonard and J. Tersoff are acknowledged for sharing the results of Ref. [17] before publication. This work was supported by the Royal Dutch Academy of Sciences (KNAW).

[1] For a recent review, see C. Dekker, Phys. Today 5, 22 (1999).

[2] J. W. G. Wildöer, L. C. Venema, A. G. Rinzler, R. E. Smalley, and C. Dekker, Nature (London) 391, 59 (1998).

[3] T. W. Odom, J. Huang, P. Kim, and C. M. Lieber, Nature (London) 391, 62 (1998).

[4] S. J. Tans, A. R. M. Verschueren, and C. Dekker, Nature (London) 393, 49 (1998).

[5] M. Dresselhaus, Phys. World 5, 18 (1996).

[6] B. I. Dunlap, Phys. Rev. B 49, 5643 (1994).

[7] Ph. Lambin, A. Fonseca, J.P. Vigneron, J. B. Nagy, and A. A. Lucas, Chem. Phys. Lett. 245, 85 (1995).

[8] L. Chico, L. X. Benedict, S. G. Louie, and M. L. Cohen, Phys. Rev. B 54, 2600 (1996).

[9] Z. Yao, H. Postma, L. Balents, and C. Dekker, Nature (London) 402, 273 (1999).

[10] M. S. Fuhrer, J. Nygård, L. Shih, M. Bockrath, A. Zettl, and P. McEuen (to be published).

[11] A. A. Farajian, K. Esfarjani, and Y. Kawazoe, Phys. Rev. Lett. 82, 5084 (1999).

[12] A. A. Odintsov and Y. Tokura, J. Low Temp. Phys. 118, 509 (2000).

[13] We expect the results to be qualitatively correct for junctions with angles $\chi<\pi / 2$. The difference $\delta U$ between the true electrostatic potential $U\left(\vec{r}_{1}-\vec{r}_{2}\right)$ for a junction with a kink $\left[\left(\vec{r}_{1} \vec{r}_{2}\right) /\left|\vec{r}_{1}\right|\left|\vec{r}_{2}\right|=-\cos \chi\right]$ and the corresponding electrostatic potential $U\left(\left|\vec{r}_{1}\right|+\left|\vec{r}_{2}\right|\right)$ for a straight junction is estimated at $\delta U / U\left(\left|\vec{r}_{1}\right|+\left|\vec{r}_{2}\right|\right) \simeq 1-$ $\left|\vec{r}_{1}-\vec{r}_{2}\right| /\left(\left|\vec{r}_{1}\right|+\left|\vec{r}_{2}\right|\right)<\chi^{2} / 8$. This amounts to $\sim 6 \%$ for the junctions with $\chi \sim 40^{\circ}$ considered in Ref. [9].

[14] In Eq. (3) $\rho$ is averaged over few atomic distances. Our approach does not describe phenomena at atomic length scale, for instance, the Friedel oscillations.

[15] We assume that the charges at the nanotube and the gate electrode are not compensated by, e.g., atmosphere ions; see L.D. Landau and E.M. Lifshitz, Electrodynamics of Continuous Media (Pergamon Press, New York, 1960), Ch. 3.

[16] P. L. McEuen, M. Bockrath, D. H. Cobden, Y.-G. Yoon, and S. G. Louie, Phys. Rev. Lett. 83, 5098 (1999).

[17] F. Léonard and J. Tersoff, Phys. Rev. Lett. 83, 5174 (1999). 